\title{
Intrasaccular Flow Disruption in Acutely Ruptured Aneurysms: A Multicenter Retrospective Review of the Use of the WEB
}

\author{
T. Liebig, C. Kabbasch, C. Strasilla, A. Berlis, W. Weber, L. Pierot, T. Patankar, X. Barreau, J. Dervin, A. Kuršumović,
} S. Rath, B. Lubicz, and J. Klisch

\begin{abstract}
BACKGROUND AND PURPOSE: Use of the WEB intra-aneurysmal flow-disruption device in unruptured wide-neck bifurcation aneurysms has proven safety and efficacy. However, ruptured aneurysms are underrepresented in existing studies. This retrospective multicenter study describes the use of the WEB in patients with a ruptured intracranial aneurysm.
\end{abstract}

MATERIALS AND METHODS: Ten centers contributed to this study. Clinical and procedural data of 47 patients with 52 aneurysms were analyzed retrospectively together with follow-up angiographies.

RESULTS: There were 37 anterior and 15 posterior circulation aneurysms with a neck size of $\geq 4 \mathrm{~mm}$ in 49 of 52 (94\%) aneurysms; 45 (87\%) aneurysms were $<10 \mathrm{~mm}$, and 2 were partially thrombosed. Successful placement of the WEB was possible in every case. Adjunctive devices were used in 8 of 52 (15\%) aneurysms. Thromboembolic events were observed in 4 of $52(8 \%)$ patients. Adverse events occurred in 15 patients with 16 aneurysms, 9 of which were potentially related to the WEB procedure ( 3 thromboembolic events, 5 protrusions, and 1 perforations; 2 perforations were caused by the wire or catheter), but none had a clinical impact. Four patients were retreated. Short-term follow-up in 25 of 39 patients revealed complete occlusion in 15 of 25 (60\%), 5 of 25 (20\%) with residual neck, and 5 of 25 (20\%) with residual aneurysm filling. Short- to midterm imaging in 9 of 25 patients revealed complete occlusion in $5(55.6 \%)$, residual neck in 2 ( $22 \%$ ), and residual aneurysm filling in 2 (22\%). Of 47 patients, $23(49 \%)$ had an mRS score of 0,1 , or 2; 13 (28\%) had an mRS score of 3 or 4 ; and none had an mRS score of 5 at discharge.

CONCLUSIONS: This retrospective series showed good procedural safety, feasibility, and stability of midterm occlusion in ruptured wide-neck bifurcation aneurysms.

ABBREVIATIONS: CLARITY = Clinical and Anatomical Results In the Treatment of Ruptured Intracranial Aneurysms; HH = Hunt and Hess; ATENA = Analysis of Treatment by Endovascular Approach of Nonruptured Aneurysms

$\mathbf{T}$ he prevalence of intracranial aneurysms in the general population ranges from $0.2 \%$ to $9.0 \% .^{1,2}$ Aneurysmal subarachnoid hemorrhage occurs at an annual incidence of 7-13 per

Received October 9, 2014; accepted after revision February 2, 2015.

From the Department of Radiology (T.L., C.K.), Universitätsklinikum Köln, Cologne, Germany; Department of Diagnostic and Interventional Radiology and Neuroradiology (C.S., J.K.), Helios General Hospital, Erfurt, Germany; Department of Diagnostic Imaging and Neuroradiology (A.B.), Klinikum Augsburg, Augsburg, Germany; Department of Neuroradiology (W.W.), Knappschaftskrankenhaus, Recklinghausen, Germany; Department of Neuroradiology (W.W.), Universitätsklinikum Knappschaftskrankenhaus Bochum, Bochum, Germany; Department of Neuroradiology (L.P.), Hôpital Maison-Blanche, Université Reims-Champagne-Ardenne, Reims, France; Leeds General Infirmary (T.P.), Leeds, United Kingdom; Department of Neuroimaging (X.B.), CHU Pellegrin, Bordeaux, France; Southern General Hospital (J.D.), Glasgow, United Kingdom; Department of Neuroradiology (A.K., S.R.), Klinikum Deggendorf, Deggendorf, Germany; and Department of Neuroradiology (B.L.) Erasme University Hospital, Brussels, Belgium.

Please address correspondence to Thomas Liebig, MD, Department of Radiology, Universitätsklinikum Köln, Kerpener Str 62, 50937 Köln, Cologne, Germany; e-mail: thomas.liebig@uk-koeln.de

- Indicates open access to non-subscribers at www.ajnr.org
100,000 person-years in most Western countries. It is associated with a high mortality rate of up to $50 \%$ and a high morbidity rate for patients admitted to the hospital. ${ }^{3,4}$ The risk of rupture increases with aneurysm size and location. ${ }^{5-9}$ The primary purpose of treating a ruptured aneurysm is to prevent recurrent hemorrhage in the acute and subacute phases. ${ }^{10}$ The preferred treatment in patients with SAH, endovascular coil occlusion may be limited by aneurysm geometry, particularly in wide-neck or broad-based aneurysms that typically necessitate the use of a stent for proper reconstruction of the neck and sufficient occlusion of the aneurysm sac. This treatment usually requires the use of antiplatelet medication, which may impose an increased risk in the presence of a recently ruptured aneurysm. ${ }^{11}$

The WEB aneurysm embolization system (WEB; Sequent Medical, Aliso Viejo, California) is a self-expanding microbraid mesh implant delivered through a microcatheter and sized to fit

http://dx.doi.org/10.3174/ajnr.A4347 

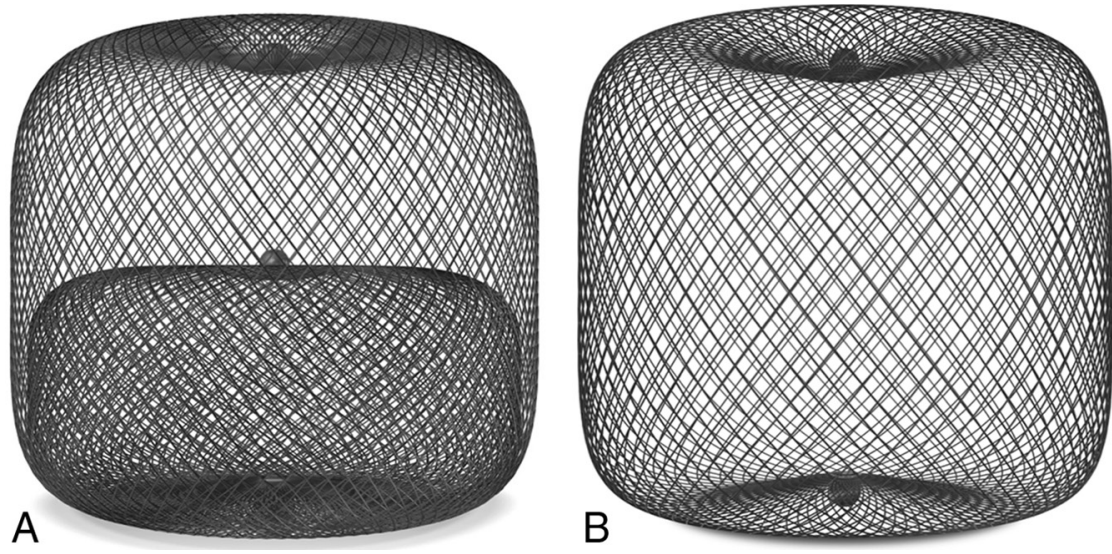

FIG 1. A, WEB-DL, a double-layer microbraid, used in 37 of the 52 aneurysms in this series. $B$, WEB-SL, a single-layer implant, used in 15 of the 52 aneurysms in this series.

and occlude an aneurysm in a single step. Two versions are available: a dual-layer type (WEB-DL), which is composed of 2 concentric cages, and a single-layer type (WEB-SL or SLS). The available sizes range from 4 to $11 \mathrm{~mm}$ in diameter and 3 to $9 \mathrm{~mm}$ in height. The mesh is designed to disrupt the flow at the neck of the aneurysm and to ultimately exclude it from the blood flow while preserving the flow in the parent artery (Fig 1). It seems to be especially suitable for broad-based bifurcation aneurysms and may eliminate the need for other reconstructive measures inside the parent artery, such as placing a stent. ${ }^{12}$ Numerous publications have described the successful use of the WEB in the treatment of unruptured wide-neck bifurcation aneurysms. ${ }^{12-14}$ Just recently, a single-center experience with the WEB in 6 ruptured aneurysms was reported, and the results were promising. ${ }^{15}$ In this retrospective multicenter case series, we describe use of the WEB in 52 aneurysms in 47 patients who presented with subarachnoid hemorrhage secondary to a ruptured intracranial aneurysm.

\section{MATERIALS AND METHODS}

A retrospective review of the medical records, radiographic imaging, and interventional reports was performed for 47 patients treated with a WEB device for a ruptured intracranial aneurysm at 1 of 10 European centers between May 2011 and December 2013. At each participating center, clinical and interventional treatment was provided per institutional standards of care. In each participating institution, the indication for treatment and technique (surgery or endovascular treatment) were decided on a case-bycase basis by a local multidisciplinary team that included neurosurgeons, neuroanesthesiologists, neurologists, and neuroradiologists. The selection of aneurysms treated with the WEB device was performed autonomously in each center by the interventional neuroradiologists according to aneurysm characteristics (ie, location, diameter, and neck size).

Permission from each patient to include their data was achieved in accordance to institutional ethical guidelines. Baseline patient and aneurysm characteristics, comorbidities, and SAH severity scores (Hunt and Hess [HH] or World Federation of Neurosurgical Societies classification) were compiled. Angiographic assessments were made at baseline, immediate postembolization, short-term (2-9 months [mean, 4.2 months]), and midterm (7-25 months [mean, 12.9 months]) time points. The immediate periprocedural hemodynamic effects and follow-up occlusion success were rated and reported by each operator as "complete," "residual filling of the neck," or "residual aneurysm filling," as previously described. ${ }^{15}$ Periprocedural medications, procedural complications, and clinical outcomes were compiled.

\section{Patient Data}

Forty-seven patients harboring a total of 52 aneurysms were evaluated. SAH severity scores ( $\mathrm{HH}$ or World Federation of Neurosurgical Societies) were available for 46 of the 47 patients. Eleven patients had an HH grade of I, 12 had an $\mathrm{HH}$ grade of II, 8 had an $\mathrm{HH}$ grade of III, 7 had an $\mathrm{HH}$ grade of $\mathrm{IV}$, and 5 had an $\mathrm{HH}$ grade of $\mathrm{V}$. The median $\mathrm{HH}$ score was II. Three patients were rated according to the World Federation of Neurosurgical Societies system; 2 patients had a grade of 1 , and 1 patient had a grade of 5 .

Three patients had 1 additional aneurysm, and 1 patient had 2 additional aneurysms. These aneurysms were treated during the same procedure with WEB devices. The anatomic locations of the ruptured aneurysms were distributed as follows: 18 in the MCA, 17 in the anterior communicating artery, 8 in the basilar artery, 6 in the posterior communicating artery, 1 in the ICA terminus, 1 in the PICA, and 1 in the pericallosal artery. In the 4 patients with multiple aneurysms treated ( 9 aneurysms total; 1 patient with 2 in the MCA and 1 in the PICA, 1 patient with 1 in the ICA terminus and posterior communicating artery, and 2 patients with 1 in the MCA and anterior communicating artery), the rupture site could not be identified with absolute certainty, and each aneurysm was treated with a WEB device. The rupture site was visible in 32 aneurysms, and in 10 it interfered with deployment of the WEB. Two of these patients were among those with periprocedural rehemorrhage, one that resulted from a microwire perforation and the other that potentially resulted from the WEB deployment itself.

Of 52 aneurysms, $10(19.2 \%)$ were $<5 \mathrm{~mm}$ in diameter, 35 $(67.3 \%)$ were $5-10 \mathrm{~mm}$ in diameter, and $7(13.5 \%)$ were $>10 \mathrm{~mm}$ in diameter. Of the 52 aneurysms, $49(94.2 \%)$ had a neck size of $\geq 4 \mathrm{~mm}$ (mean, $4.7 \mathrm{~mm}$ ). Two of the large aneurysms were partially thrombosed.

\section{Procedural Data}

Two treatments were performed as one staged procedure; one required additional coiling of a remnant during a control angiogram within 24 hours, and the other was planned as a 2-step occlusion, first of the dome, including the rupture site, followed 4 days later by stent-assisted coil occlusion with reconstruction of the neck under dual antiplatelet medication.

For 34 of the aneurysms, the index procedure was performed with the patient having been given a full bolus of IV heparin (5000 IU); 9 aneurysms were treated with a reduced bolus (2500 IU, all in the same center); and 9 were treated without heparin. Heparin was maintained at partial thromboplastin time levels of $40-50$ 
Table 1: Periprocedural events

\begin{tabular}{|c|c|c|c|c|c|}
\hline Patient No. & AN Location & Complication & Device Related & Outcome (mRS Score) & Clinical Consequence \\
\hline 1 & MCA & TE & Yes & 0 at $8 \mathrm{mo}$ & None \\
\hline 2 & MCA & TE & Yes & 3 (same as before $\mathrm{SAH}$ ) & None \\
\hline 3 & PcomA & TE & Yes & 0 at $3 \mathrm{mo}$ & None \\
\hline 4 & BA & TE & No & 4 at discharge $\mathrm{e}^{\mathrm{a}}$ & None \\
\hline \multirow[t]{2}{*}{5} & MCA & $\mathrm{TE}$ & No & 3 at $2 \mathrm{mo}$ & Yes \\
\hline & AcomA & AN perforation & No & & Yes \\
\hline 6 & Pericallosal artery & AN perforation & Yes & 0 at $1 \mathrm{wk}$ & None \\
\hline 7 & MCA & AN perforation & No & 6 from initial SAH & Unknown ${ }^{\text {b }}$ \\
\hline 8 & AcomA & Induced spasm & Unknown & 6 from initial SAH & Unknown $^{b}$ \\
\hline 9 & MCA & Induced spasm & Unknown & Unknown & Unknown \\
\hline 10 & PcomA & Infarction & No & 2 at $6 \mathrm{mo}$ & Transient \\
\hline 11 & AcomA & WEB protrusion & Yes & 0 at $6 \mathrm{mo}$ & None \\
\hline 12 & BA & WEB protrusion & Yes & 0 at $8 \mathrm{mo}$ & None \\
\hline 13 & MCA & WEB protrusion & Yes & 0 at 3 mo & None \\
\hline 14 & AcomA & WEB protrusion & Yes & 0 at $25 \mathrm{mo}$ & None \\
\hline 15 & rMCA, IMCA, Dissect Vert & rMCA branch partial occlusion & Yes & 0 at 3 wk & None \\
\hline
\end{tabular}

Note:-AcomA indicates anterior communicating artery; AN, aneurysm; BA, basilar artery; Dissect Vert, dissecting vertebral; IMCA, left MCA; PcomA, posterior communicating artery; rMCA, right MCA; TE, thromboembolic event.

${ }^{a}$ Clinical outcome related to ventriculitis.

${ }^{\mathrm{b}}$ Death during hospitalization secondary to the $\mathrm{SAH}$, not during the procedure.

seconds for 48 hours in 5 patients from one center. Low-molecular-weight heparin was maintained in 22 patients ( 21 from 1 center). Only 1 patient was known to be on aspirin before the treatment. Twenty-three patients received 250-500 $\mathrm{mg}$ of aspirin during the intervention, usually before introduction of the WEB device. Glycoprotein IIb/IIIa inhibitors were used to counteract periprocedural thrombus formation: eptifibatide (Integrilin) in 2 cases of visible clot and abciximab (ReoPro) in 1 case of WEB protrusion. Postprocedural antiaggregation was maintained continuously in 27 patients (75-100 mg aspirin/day), and 7 of 27 received additional clopidogrel $(75 \mathrm{mg} /$ day $)$ for 12 weeks. Eight patients who were kept on posttreatment antiplatelet medication were among those who had either periprocedural thrombus formation or postapplication protrusion of the WEB as the reason to keep up the medication. Another 19 patients were kept on a single antiplatelet medication without evident procedural reason (per the institutional standard of care). One patient with a periprocedural clot formation was treated with mechanical clot extraction by means of a Solitaire stent (Covidien, Irvine, California) and was administered a single 500-mg dose of aspirin, but no continuous medication was given.

Most procedures were performed with 6F access catheters/ long sheaths, and 17 of 52 aneurysms were treated with intermediate catheters in addition to the dedicated microcatheters (32 VIA or VIA PLUS [Sequent Medical], 20 other catheters of $\geq 0.027$ inches). Thirty-seven aneurysms were treated with a duallayer device that was composed of 2 cages of 144 woven nitinol strands, and 15 aneurysms were treated with single-layer devices in which the number of nitinol strands varied from 144 to 216 (on the basis of device diameter).

\section{RESULTS}

Eight aneurysms were treated with additional devices ( 4 with stent only, 1 with coils only, 1 with coils and a stent, 1 with coils and a balloon, 1 with a balloon). The reasons for adjunctive treatments were planned staged procedure (1 case), to complete neck closure or sac filling ( 2 cases), to treat preaneurysm stenosis ( 1 case), and to correct device protrusion (4 cases).
Treatment durations ranged from 15 to 193 minutes (average, 68 minutes). Treatment durations for 4 of the treatments with WEB placement in multiple aneurysms ranged from 67 to 195 minutes.

\section{Adverse Events}

Periprocedural adverse events were reported for 15 patients during the treatment of 16 aneurysms, including 1 patient with 2 complications. Among those who underwent these procedures, 10 patients had no clinical sequelae and were discharged with an mRS score of $<2$ or a score equal to that before the initial SAH (Table 1).

Thirty-six aneurysms were treated without adverse events. In a maximum of 12 of 16 complicated treatments, the adverse event could possibly be attributed to the actual delivery of the WEB device. However, 3 of those were caused by the guidewire ( 1 perforation) or the microcatheter probing (1 perforation and 1 induced spasm).

Four events were thromboembolic in nature and required antiplatelet medication; 1 received eptifibatide, 1 received eptifibatide and additional aspirin and clopidogrel, 1 clot progressed despite $500 \mathrm{mg}$ of intravenous aspirin and was removed successfully with a Solitaire stent, and in 1 case there was no information with regard to treatment. None of these patients had clinical sequelae.

Four aneurysms exhibited angiographic protrusion of the WEB that necessitated the use of a stent and, in 1 case, the additional use of IV antiplatelet drugs.

Three aneurysms were perforated during the treatment. However, only 1 case presented evidence of an additional SAH on postprocedural CT. This SAH might have occurred during the deployment of the device itself, and there was no clinical impact on the patient. The other 2 perforations were caused by a wire or a catheter.

There was no delayed bleeding in any of the patients treated with a WEB device.

Angiographic Results. An immediate postprocedural hemodynamic effect was achieved in 44 of 52 (84.6\%) aneurysms. Twenty 
Table 2: Nonfatal complications during hospitalization

\begin{tabular}{|c|c|c|c|c|}
\hline Patient No. & AN Location & Type of Event & SAH Related & Outcome (mRS Score) \\
\hline 1 & AcomA & Vasospasm & Yes & 0 at discharge \\
\hline 2 & MCA & Vasospasm on day 14 & Yes & 2 at $6 \mathrm{mo}$ \\
\hline 3 & MCA & Vasospasm on day 7 & Yes & 0 at discharge \\
\hline 4 & BA & Vasospasm & Yes & 4 at $12 \mathrm{mo}$ \\
\hline 5 & AcomA & Infarct on MRI & Yes & 2 at $6 \mathrm{mo}^{\mathrm{a}}$ \\
\hline 6 & MCA & Stroke on MRI & No & 0 at discharge \\
\hline 7 & BA & Ventriculitis & Yes & 4 at discharge \\
\hline 8 & AcomA & Rehemorrhage; additional treatment with coils & No & 0 at $6 \mathrm{mo}$ \\
\hline 9 & BA & New infarct on day 3 with vasospasm and stenosis visible & Yes & 0 at $8 \mathrm{mo}$ \\
\hline 10 & MCA & Stroke on MRI & No & 3 at discharge \\
\hline 11 & rMCA, IMCA, Dissect Vert & Brain stem edema on day 23 & No & 0 at 3 wk \\
\hline
\end{tabular}

Note:-AcomA indicates anterior communicating artery; AN, aneurysm; BA, basilar artery; Dissect Vert, dissecting vertebral; IMCA, left MCA; rMCA, right MCA.

${ }^{a}$ Clinical status restored to pre-SAH value in a patient with multiple sclerosis.

(38.5\%) aneurysms were completely occluded, 15 (28.8\%) only showed contrast in the proximal compartment of the implant, and another $9(17.3 \%)$ showed at least occlusion of the aneurysm dome beyond the implant. In 1 patient, a coil was also used within 24 hours to occlude an angiographic remnant outside the WEB that was not covered by the WEB itself after its initial placement. Of the 8 aneurysms that did not even partially occlude, 6 at least showed delay of the contrast washout until the venous phase, 1 even after the venous phase.

Imaging performed in 25 of 39 patients still alive at short-term follow-up (mean, 4 months [range, 2-9 months]; 21 performed with DSA, 4 performed with MRA) revealed complete occlusion of the aneurysm in 15 of 25 (60\%) cases, 5 of 25 (20\%) had residual filling of the neck, and 5 of 25 (20\%) showed residual filling of the aneurysm. Imaging performed on 9 of 25 patients at midterm (mean, 13 months [range, 7-36 months]; all DSA) revealed complete occlusion of the aneurysm in 5 of $9(55.6 \%)$ cases, 2 of 9 $(22.2 \%)$ had residual neck, and 2 of $9(22.2 \%)$ had residual aneurysm filling. In the midterm follow-ups, none of the implants themselves showed filling with contrast. There was no conceivable difference between the single- and dual-layer implants. Among the 20 aneurysms with immediate complete occlusion, 12 were treated with a dual-layer device, and 8 were treated with a singlelayer device. None of the aneurysms that exhibited residual filling of the sac on midterm follow-up had been treated with a singlelayer WEB.

Four patients required endovascular retreatment. In 1 patient, DSA was performed because of neurologic worsening on the day of the initial treatment; it was decided to treat this residual aneurysm with coils. One patient required endovascular retreatment with coils 1 month after the initial procedure. One patient had 3 aneurysms treated with a WEB device initially, and additional endovascular treatment was required because of a dissecting V4 aneurysm with coils and Y-stent placement 3 weeks later. One patient required retreatment with coils and a stent 3 months later for aneurysm regrowth caused by integration of the WEB into an intra-aneurysmal thrombus. All remnants or recurrences were treated by endovascular means without additional adverse events.

Clinical Outcome Data. Of 47 patients, 23 were discharged as independent survivors with an mRS score of 0,1 , or $2 ; 13$ patients had an mRS score of 3 or 4; and none had an mRS score of 5 . Eight patients died during their hospital stay, 5 as a result of the $\mathrm{SAH}$ and 3 for other reasons ( 1 from rehemorrhage 3 hours after treat- ment, probably as a result of dissection from another aneurysm; 1 from acute respiratory distress syndrome; and 1 from vasospasm and infarction of all territories). For 3 patients, the postprocedural mRS score was not recorded.

Nineteen patients experienced a complication during their hospital stay. Of these 19 complications, 16 were related to the initial SAH (eg, vasospasm, new infarction on follow-up imaging, or ventriculitis after insertion of an extraventricular drainage). Three patients experienced postprocedural hemorrhage during their hospital stay: 1 intraparenchymal hemorrhage resulted from external ventricular drainage, 1 aneurysm was insufficiently occluded and was coiled within 24 hours after clinical worsening of the patient, which may have been caused by rehemorrhage, and 1 patient experienced rupture of an additional aneurysm 3 hours after the treatment of the index aneurysm with a WEB. This rupture was of a dissecting aneurysm that was treated with coils but had a fatal outcome. The dissecting aneurysm was identified as the source of rehemorrhage by autopsy.

Of 16 complications that were reportedly related to the intraoperative device and/or the procedure, 9 occurred in patients who were discharged with an mRS score of $>2$; one of these 9 patients already had a pretreatment mRS score of 3 and was discharged unchanged. Two of these 9 patients died, one of whom had a guidewire perforation during the treatment; 5 of 9 had an mRS score of 3 or 4 , and no information was available for the other 2 patients.

Of the 30 patients available for midterm follow-up, 27 were independent. Of the remaining 3 , two had maintained their initial status, and 1 had progressed from an mRS score of 1 at discharge to an mRS score of 4 after an episode of intraparenchymal hemorrhage after tPA administration for pulmonary artery thrombosis (Table 2).

\section{DISCUSSION}

Endovascular coil occlusion has been proven to increase the number of patients with good clinical outcome over that of those who undergo surgical clipping after aneurysmal subarachnoid hemorrhage. ${ }^{16}$ However, it has 2 potential drawbacks. First, coil occlusion does not immediately and completely exclude the aneurysm from the bloodstream ${ }^{17}$; presumably, this is one explanation for the slightly higher rate of early rehemorrhage after coiling in the International Subarachnoid Aneurysm Trial. ${ }^{16}$ Second, coiled aneurysms have a greater tendency to recur particularly if they are 


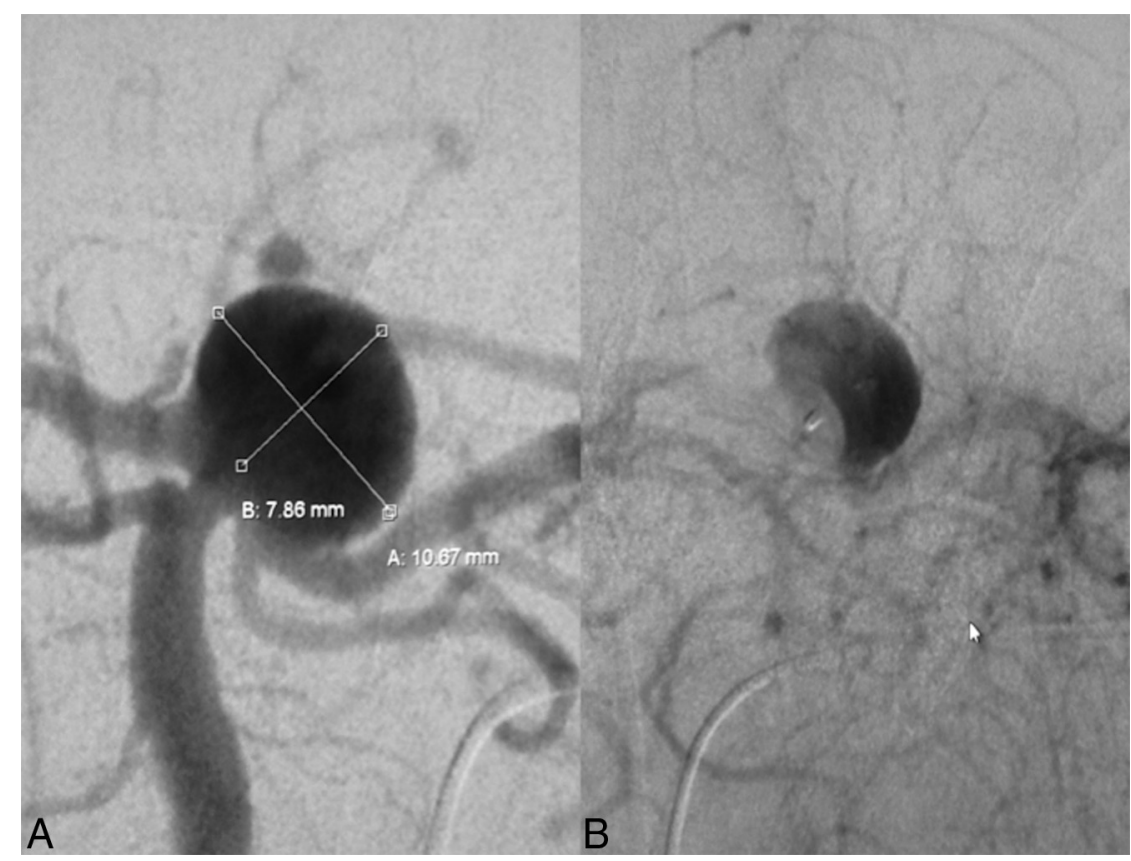

FIG 2. Ruptured basilar tip aneurysm before $(A)$ and immediately after $(B)$ deployment of the WEB device. After the WEB is placed, no contrast filling can be seen inside the dome, including the bleb that is presumed to be the rupture site; furthermore, there is stasis of contrast inside the WEB beyond the venous phase, indicating sufficient hemodynamic decoupling.
Clinical and Anatomical Results In the Treatment of Ruptured Intracranial Aneurysms (CLARITY) study. ${ }^{21,22} \mathrm{Al}$ though the percentage of broad-based aneurysms was much higher in the $\mathrm{Pa}$ pagiannaki et al series ( $85.9 \%$ vs $9.1 \%$ in CLARITY), the results for peri-interventional rupture and thromboembolic events were comparably low, and the rate of adequate occlusion was even higher with WEB than in CLARITY. Despite these observations, there is still a lack of evidence regarding the treatment results for WEB in ruptured aneurysms so far; in the Papagiannaki et al study, only 4 aneurysms (4.6\%) were ruptured.

In the present series of 52 ruptured aneurysms, the technical success was $100 \%$. However, failed attempts to place a WEB device or even the catheter and the subsequent choice of other treatment algorithms were not specifically recorded.

A limitation of this novel implant, which can be attributed to the larger microcatheter needed and greater rigidity

large, broad-based, partially thrombosed, or ruptured. Furthermore, standard coil occlusion may be limited or unsuitable because of aneurysm geometry. Broad-based aneurysms especially may require the placement of a stent to remodel the parent artery, which by itself necessitates the use of antiplatelet medication. Thus, an endovascular treatment alternative that may be used safely for broad-based aneurysms without the need for additional implants in the bloodstream, yet offers efficacious aneurysm occlusion and prevents rehemorrhage, seems to be desirable, particularly for ruptured aneurysms. The purpose of this study was to evaluate the use of a relatively novel intra-aneurysmal microbraid implant, the WEB device, in ruptured aneurysms. In nonruptured aneurysms, the WEB has been described in a number of published series to be both safe and efficacious. ${ }^{12-14}$ Because of its conceptual difference from standard coil occlusion, a potential yet unproven advantage of the WEB is faster hemodynamic separation of the aneurysm; immediate stasis is often seen after its deployment (Fig 2). The absence of overlapping radiopaque material might enable accurate positioning at the level of the neck, even in broad-based aneurysms. At the same time, stronger and larger microcatheters and the acute tip of the implant at the early stage of deployment and before unfolding may impose an increased risk for periprocedural rupture. Finally, nitinol coils have a potentially higher thrombogenicity when compared with the very inert platinum coils. ${ }^{18}$ Therefore, thromboembolic events are of special concern, accepting that subarachnoid hemorrhage is known to induce a hypercoagulative state. ${ }^{19}$

Recently, a multicenter study by Papagiannaki et $\mathrm{al}^{20}$ of 87 aneurysms (mostly unruptured) in 85 patients was published, and the results were favorable for the WEB when compared with those of coil occlusion in the Analysis of Treatment by Endovascular Approach of Nonruptured Aneurysms (ATENA) trial and the during the initial delivery when compared with those of coils, is the fact that in 10 of 52 aneurysms, the operator felt that the implant interfered with the rupture site during its delivery. However, only in one such case could a periprocedural rupture (seen as an increase in the extent of subarachnoid blood on postprocedural CT) potentially have been attributed to the WEB delivery and was of no clinical consequence whatsoever (patient with an mRS score of 0 on follow-up). Two other complications included microguidewire and microcatheter perforations, which occurred before each implant was advanced.

Because of the nature of subarachnoid hemorrhage, the overall clinical outcome in our series was worse than that in the Papagiannaki et al series. Eight patients died during hospitalization, and another 11 patients experienced SAH-related complications such as severe vasospasm leading to infarction or ventriculitis after external ventricular drainage. However, after the acute phase, the results were relatively good. Of the 30 patients available for midterm follow-up, 27 were independent survivors (mRS scores, $0-2$ ). Of the remaining 3 patients, 2 have remained at their pretreatment status, and only 1 had progressed from an mRS score of 1 at discharge to an mRS score of 4 after an episode of intracranial hemorrhage after tPA administration for pulmonary artery thrombosis 7 months after the index procedure. Most important is that there was no late rerupture or late ischemia that could have been attributed to the WEB. All rehemorrhages occurred during the acute or subacute phase and could be explained by either periprocedural perforation with the wire or the catheter/WEB or incomplete occlusion of the aneurysm, leaving the potential rupture site unprotected by the implant. The midterm occlusion rates compare favorably to those of other series of ruptured aneurysms, such as CLARITY. Here, adequate occlusion was observed in 419 of $517(81.0 \%)$ aneurysms, and an aneurysm remnant was found 
in 98 of $517(19.0 \%)$ aneurysms at midterm follow-up. In our series, only 4 aneurysms required additional treatment. One was a dissecting PICA vertebral aneurysm, initially misjudged as a PICA aneurysm and later treated with an additional stent and coils. Another procedure was staged with a WEB in the acute phase followed by surgical clipping. The remaining 2 aneurysms were a residual filling at the neck at 1 month treated with coils and regrowth of a partially thrombosed aneurysm that had been treated with 2 WEB devices, which both became integrated into the clot. The recurrence was treated with a stent and coils. Furthermore, the results of our series cannot really be compared with those of CLARITY, because 49 of the 52 ruptured aneurysms in our study had a neck size of $\geq 4 \mathrm{~mm}$ versus the only $9.1 \%$ broad-based ruptured aneurysms in CLARITY.

The $30.8 \%$ ( 16 of 52 ) rate of procedural events may seem high at first. However, the operators were ask to record all procedural events, including those without clinical sequelae, such as transient thrombus formation, aggravation of vasospasm induced by wire and catheter probing, and protrusion of the WEB. When only events that effectively resulted in clinical worsening or prolonged hospital stay were rated, this figure was reduced to 3 of 52: one aneurysm perforated with the guidewire, one thromboembolic event, and the aforementioned rehemorrhage in a case in which the presumed site of rupture was not covered with the WEB during the first treatment. One additional patient exhibited induction or aggravation of vasospasm caused by probing with the microcatheter; this patient died as a result of SAH sequelae. Thus, the overall rate of procedural complications with clinical worsening can be extrapolated to parallel that in the aforementioned series of nonruptured aneurysms treated with a WEB device (13\% in the Papagiannaki et al study). The rate of intraoperative rupture (5.7\% vs $1.3 \%$ ) was slightly higher than that in the Papagiannaki et al study and than those of ATENA (2.6\%) and CLARITY $(3.7 \%)$. The small sample size in our study did not allow for a validated comparison. The same limitation exists for the rate of thromboembolic events, mainly comprising thrombus formation on the implant itself that was more or less equal to that in the Papagiannaki et al series (9.6\% vs 7.6\%, respectively). Morbidity and mortality rates are difficult to calculate for our group of patients with SAH, because most of the deaths and poor outcomes could be attributed to the SAH itself. There were only 3 procedural events that most likely led to clinical consequences, only one of which was related to the implant itself (thromboembolic branch occlusion). For comparison, the treatment morbidity and mortality rates were $1.3 \%$ and $0.0 \%$ in the Papagiannaki et al series, $1.7 \%$ and $1.4 \%$ in ATENA, and $3.7 \%$ and $1.5 \%$ in CLARITY, respectively. $^{20-22}$

Between the 2 different types of WEB devices (single- or duallayer), not many differences could be found. First, because of the later introduction of the WEB-SL, all treatments before June 2013 were performed with the WEB-DL. Thus, only 15 aneurysms were treated with a single-layer device. With the introduction of the single-layer WEB, the question of whether it would be similarly effective in flow disruption arose. In our series, we could not find a difference in efficacy between the 2 designs, neither with regard to the immediate angiographic result nor to long-term occlusion. The absolute numbers were actually in favor of the single-layer WEB device. Immediate complete occlusion was recorded for 12 of 37 WEB-DL and 8 of 15 WEB-SL devices. None of the aneurysms with residual filling of the sac on midterm follow-up was treated with a single-layer WEB. We interpret this trend as an indication that the flow-disrupting effect of the single-layer system most likely is at least as good as that with the dual-layer WEB; this, however, remains to be proved in a larger comparative study.

With regard to thromboembolic potential, which certainly plays an important role in the hypercoagulative state during acute $\mathrm{SAH}$, our results were also in favor of the single-layer version. Only 1 thromboembolic event was recorded for the WEB-SL device, and it consisted of local clot formation that could be removed by means of stent-retriever thrombectomy; all the other thromboembolic events occurred with the WEB-DL device. Finally, protrusion of the implant seemed to be an issue that depended on aneurysm geometry, because protrusion occurred in aneurysms $\leq 6 \mathrm{~mm}$ in diameter. More important is that these aneurysms had a neck-to-height ratio of $>1$, meaning that these aneurysms were not only small but also shallow. With regard to protrusion, the type of implant did not seem to make a difference. Recent developments of the WEB with enhanced visibility and new angiographic visualization techniques seem to enable us to reliably detect and avoid protrusion as a potential source of adverse events in the future. ${ }^{23}$ There was 1 aneurysm with partial thrombosis of the sac that had recurred at 3 months. Hope that the greater and more even surface of the WEB may sufficiently prevent migration into the clot, regularly seen with bare coils, is not warranted at the moment. Overall, the WEB provided safe aneurysm occlusion without necessitating additional stent placement in $90 \%$ of the patients. Periprocedural double-antiplatelet treatment could have been avoided in $87 \%$ of the patients and single-antiplatelet treatment in $50 \%$ of the patients in the acute phase of SAH. Although the follow-up period was shorter in our cohort, the rate of target aneurysm rerupture (1 of 52 [2\%]) is comparable with that found in larger trials: $4.2 \%$ in the International Subarachnoid Aneurysm Trial for 1 year and $4.5 \%$ in a series of 44 ruptured wide-neck aneurysms treated with a stent and coils. ${ }^{24}$

\section{CONCLUSIONS}

The WEB intra-aneurysmal flow disruptor can be used in ruptured aneurysms with safety and efficacy equal to those shown in previous experiences gathered with use of the WEB device in nonruptured aneurysms. Comparison with previous series of ruptured aneurysms was compromised by the fact that in most cases in our series, the aneurysms were broad-based. This specific geometry in combination with acute rupture renders the rate of necessary retreatments favorably in comparison with that of most series of coil occlusion. Partial thrombosis is still an unsolved issue, because the WEB may become integrated into a clot over time. The single-layer version performed as well as the dual-layer device in our series, with absolute numbers in favor of the WEBSL, particularly with regard to thromboembolism. Protrusion of the implant is a potential problem that more likely may occur in small and shallow aneurysms, which is a limitation that will probably be overcome with an enhanced-visibility version of the braid and new angiographic imaging modalities. Overall, the WEB seems to be a very suitable treatment option for ruptured aneurysms, even if they are broad-based. Concerns that the conceptual 
differences between the WEB device and coil occlusion may be associated with an increased risk of rehemorrhage are not warranted by the results of this series.

Disclosures: Thomas Liebig-UNRELATED: Consultancy: Consulting and proctoring for Sequent Medical, Acandis, MicroVention, Concentric/Stryker, and eV3. Ansgar Berlis-RELATED: Consulting Fee or Honorarium: Consultancy and proctoring agreements with Sequent Medical; UNRELATED: Payment for Lectures (including service on speakers bureaus): Penumbra and Stryker. Werner Weber-RELATED: Fees for Participation in Review Activities such as Data Monitoring Boards, Statistical Analysis, Endpoint Committees, and the Like: WEB CAST study*; UNRELATED: Consultancy: Proctor for WEB cases in other institutions. Laurent Pierot-RELATED: Consulting Fee or Honorarium: Sequent Medical; UNRELATED: Consultancy: Codman, Covidien/eV3, MicroVention, and Stryker. Tufail Patankar-UNRELATED: Other: Proctor for WEB device. Xavier Barreau—RELATED: Fees for Participation in Review Activities such as Data Monitoring Boards, Statistical Analysis, Endpoint Committees, and the Like: Sequent Medical*; UNRELATED: Consultancy: Sequent Medical. James Dervin-RELATED: Support for Travel to Meetings for the Study or Other Purposes: Sequent Medical, Comments: WEB training course in Magdeburg, Germany, April 2013, travel and accommodation paid by Sequent Medical; UNRELATED: Consultancy: Sela Medical, Comments: Proctor Sela Medical $€ 1300$, January 2013; Travel/Accommodations/Meeting Expenses Unrelated to Activities Listed: Covidien and Sela Medical, Comments: Travel and accommodation and registration for the following meetings from Covidien: LINC meeting, Paris, June 2013; ESMINT meeting, Nice, France, September 2014; ALICE meeting, Essen, Germany, May 2012; and Neurovascular course, Tel Aviv, December 2013. Boris Lubicz-UNRELATED: Consultancy: MicroVention and Codman. Joachim Klisch-UNRELATED: Consultancy: Sequent Medical. * *Money paid to the institution.

\section{REFERENCES}

1. Rinkel GJ, Djibuti M, Algra A, et al. Prevalence and risk of rupture of intracranial aneurysms: a systematic review. Stroke 1998;29:251-56

2. Jeon TY, Jeon P, Kim KH. Prevalence of unruptured intracranial aneurysm on MR angiography. Korean J Radiol 2011;12:547-53

3. Linn FH, Rinkel GJ, Algra A, et al. Incidence of subarachnoid hemorrhage: role of region, year, and rate of computed tomography: a meta-analysis. Stroke 1996;27:625-29

4. de Rooij NK, Linn FH, van der Plas JA, et al. Incidence of subarachnoid haemorrhage: a systematic review with emphasis on region, age, gender and time trends. J Neurol Neurosurg Psychiatry 2007; 78:1365-72

5. Weir B, Disney L, Karrison T. Sizes of ruptured and unruptured aneurysms in relation to their sites and the ages of patients. $J \mathrm{Neu}$ rosurg 2002;96:64-70

6. International Study of Unruptured Intracranial Aneurysms Investigators. Unruptured intracranial aneurysms - risk of rupture and risks of surgical intervention. $N$ Engl J Med 1998;339:1725-33

7. Wermer MJ, van der Schaaf IC, Algra A, et al. Risk of rupture of unruptured intracranial aneurysms in relation to patient and aneurysm characteristics: an updated meta-analysis. Stroke 2007;38: 1404-10

8. Jeong YG, Jung YT, Kim MS, et al. Size and location of ruptured intracranial aneurysms. J Korean Neurosurg Soc 2009;45: $11-15$

9. Asari S, Ohmoto T. Natural history and risk factors of unruptured cerebral aneurysms. Clin Neurol Neurosurg 1993;95:205-14
10. Johnston SC, Dowd CF, Higashida RT, et al; CARAT Investigators. Predictors of rehemorrhage after treatment of ruptured intracranial aneurysms: the Cerebral Aneurysm Rerupture After Treatment (CARAT) study. Stroke 2008;39:120-25

11. Meckel S, Singh TP, Undrén P, et al. Endovascular treatment using predominantly stent-assisted coil embolization and antiplatelet and anticoagulation management of ruptured blood blister-like aneurysms. AJNR Am J Neuroradiol 2011;32:764-71

12. Lubicz B, Klisch J, Gauvrit JY, et al. WEB-DL endovascular treatment of wide-neck bifurcation aneurysms: short- and midterm results in a European study. AJNR Am J Neuroradiol 2014;35:432-38

13. Pierot L, Liebig T, Sychra V, et al. Intrasaccular flow-disruption treatment of intracranial aneurysms: preliminary results of a multicenter clinical study. AJNR Am J Neuroradiol 2012;33:1232-38

14. Pierot L, Klisch J, Cognard C, et al. Endovascular WEB flow disruption in middle cerebral artery aneurysms: preliminary feasibility, clinical, and anatomical results in a multicenter study. Neurosurgery 2013;73:27-34; discussion 34-35

15. Caroff J, Mihalea C, Dargento F, et al. Woven Endobridge (WEB) device for endovascular treatment of ruptured intracranial wideneck aneurysms: a single-center experience. Neuroradiology 2014; 56:755-61

16. Molyneux AJ, Kerr RS, Yu LM, et al; International Subarachnoid Aneurysm Trial (ISAT) Collaborative Group. International subarachnoid aneurysm trial (ISAT) of neurosurgical clipping versus endovascular coiling in 2143 patients with ruptured intracranial aneurysms: a randomised comparison of effects on survival, dependency, seizures, rebleeding, subgroups, and aneurysm occlusion. Lancet 2005;366:809-17

17. Sorteberg A, Sorteberg W, Rappe A, et al. Effect of Guglielmi detachable coils on intraaneurysmal flow: experimental study in canines. AJNR Am J Neuroradiol 2002;23:288-94

18. Mullan S, Raimondi AJ, Dobben G, et al. Electrically induced thrombosis in intracranial aneurysms. J Neurosurg 1965;22:539-47

19. Itoyama Y, Fujioka S, Takaki S, et al. Significance of elevated thrombin-antithrombin III complex and plasmin-alpha 2-plasmin inhibitor complex in the acute stage of nontraumatic subarachnoid hemorrhage. Neurosurgery 1994;35:1055-60

20. Papagiannaki C, Spelle L, Januel AC, et al. WEB intrasaccular flow disruptor-prospective, multicenter experience in 83 patients with 85 aneurysms. AJNR Am J Neuroradiol 2014;35:2106-11

21. Cognard C, Pierot L, Anxionnat R, et al; Clarity Study Group. Results of embolization used as the first treatment choice in a consecutive nonselected population of ruptured aneurysms: clinical results of the Clarity GDC study. Neurosurgery 2011;69:837-41; discussion 842

22. Pierot L, Spelle L, Vitry F; ATENA Investigators. Immediate clinical outcome of patients harboring unruptured intracranial aneurysms treated by endovascular approach: results of the ATENA study. Stroke 2008;39:2497-504

23. Caroff J, Mihalea C, Neki H, et al. Role of C-arm VasoCT in the use of endovascular WEB flow disruption in intracranial aneurysm treatment. AJNR Am J Neuroradiol 2014;35:1353-57

24. Alurkar A, Karanam LS, Nayak S, et al. Stent-assisted coiling in ruptured wide-necked aneurysms: a single-center analysis. Surg Neurol Int 2012;3:131 